\title{
Specific chemical reactivities of spatially separated 3-aminophenol conformers with cold $\mathrm{Ca}^{+}$ions
}

\author{
Yuan-Pin Chang, ${ }^{1 \dagger}$ Karol Długołęcki, ${ }^{1}$ Jochen Küpper ${ }^{1,2,3 *}$, \\ Daniel Rösch, ${ }^{4 \dagger}$ Dieter Wild, ${ }^{4}$ Stefan Willitsch ${ }^{4 *}$ \\ ${ }^{1}$ Center for Free-Electron Laser Science, DESY, \\ Notkestrasse 85, 22607 Hamburg, Germany \\ ${ }^{2}$ The Hamburg Center for Ultrafast Imaging, University of Hamburg, \\ Luruper Chaussee 149, 22761 Hamburg, Germany \\ ${ }^{3}$ Department of Physics, University of Hamburg, \\ Luruper Chaussee 149, 22761 Hamburg, Germany \\ ${ }^{4}$ Department of Chemistry, University of Basel, \\ Klingelbergstrasse 80, 4056 Basel, Switzerland \\ ${ }^{\dagger}$ These authors contributed equally to this work. \\ *To whom correspondence should be addressed; e-mail: \\ jochen.kuepper@cfel.de, stefan.willitsch@unibas.ch
}

\begin{abstract}
Many molecules exhibit multiple rotational isomers (conformers) that interconvert thermally and are difficult to isolate. Consequently, a precise characterization of their role in chemical reactions has proven challenging. We have probed the reactivity of specific conformers using an experimental technique based on their spatial separation in a molecular beam by electrostatic deflection. The separated conformers react with a target of Coulomb-crystallized ions in a trap. In the reaction of $\mathrm{Ca}^{+}$with 3 -aminophenol, we find a twofold larger rate constant for the cis- compared to the trans-conformer (differen-
\end{abstract}




\section{tiated by the $\mathrm{O}-\mathrm{H}$ bond orientation). This result is explained by conformer- specific differences in the long-range ion-molecule interaction potentials. Our approach demonstrates the possibility of controlling reactivity through selec- tion of conformational states.}

Recent progress in the cooling, manipulation and control of isolated molecules in the gas phase (1-4) has paved the way for the study of chemical processes at high levels of sensitivity, selectivity, and detail. Methods for the slowing and merging of supersonic molecular beams have enabled precise characterizations of the role of the collision energy and of the molecular quantum state in scattering and reactive processes (5-7). Recent experiments with trapped, translationally cold molecules and ions have provided insights into the quantum dynamics of chemical reactions (8) and the subtleties of intermolecular interactions (9). These experiments have thus far been restricted to reactions involving atoms or small molecules with simple geometric and quantum structures. The vast majority of molecules, however, possess a plethora of internal degrees of freedom that are challenging to probe independently. In particular, polyatomic molecules usually exhibit many rotational isomers (conformers) that interconvert with low thermal barriers through rotations about covalent bonds. Recent years have seen impressive progress in the spectroscopic characterization of specific conformations (10-12), their photoinduced isomerization $(13,14)$, and the characterization of conformer-specific photodissociation dynamics $(15,16)$. However, studies of specific conformational effects in bimolecular reactions are still sparse $(17,18)$, in particular in the gas phase where the highest degree of control and therefore insight into fundamental reaction mechanisms can be gained.

Here, we introduce a distinct approach for the study of conformational effects and conformation-dependent reactivities, i. e., rate constants, in bimolecular reactions. Our method exploits the spatial separation of different conformers using inhomogeneous electric fields $(19,20)$. Internally cold molecules in a beam are dispersed by an electrostatic deflector and are directed 
towards the reaction volume. There, they interact with laser cooled ions in a Coulomb crystal, i. e., an ordered structure of translationally cold ions in a trap (4). The Coulomb crystal constitutes a tightly localized, stationary reaction target much smaller in extension than the conformationally separated regions of the beam so that chemical reactions can be studied selectively with isolated conformers.

We studied the reaction of individual conformers of 3-aminophenol (AP) with Coulombcrystallized $\mathrm{Ca}^{+}$ions to probe conformation-dependent reactivities in a prototypical reaction between an organic molecule and a metal ion in the gas phase. Reactions of this type are of interest, e.g., in the context of bond activation in catalysis $(21,22)$ and elucidating interactions between metal ions and biomolecular building blocks (23). Fig. 1 shows a schematic of the experimental setup. The vapor above a sample of AP heated to $145{ }^{\circ} \mathrm{C}$ was entrained in a pulsed supersonic expansion of neon at a stagnation pressure of 34 bar. AP exhibits two distinct molecular conformations cis and trans that differ in the relative orientation of the $\mathrm{O}-\mathrm{H}$ bond with respect to the $\mathrm{NH}_{2}$-group. The molecular geometries are depicted in Fig. 2 A. The barrier to interconversion by rotation of $\mathrm{OH}$ about the $\mathrm{C}-\mathrm{O}$ bond is $\approx 1500 \mathrm{~cm}^{-1}(24)$. Both conformers have significantly different electric dipole moments of $0.77 \mathrm{D}$ and $2.33 \mathrm{D}$ for the trans and cisspecies, respectively, giving rise to their distinct Stark interactions with an electric field (25).

In the expansion, the AP molecules were adiabatically cooled to a population ratio of the cis and trans conformers in the molecular beam of approximately $1: 4$ and a rotational temperature of $T_{\text {rot }}=1.1 \mathrm{~K}$. Thus, over $99 \%$ of the population of both conformers was confined to the lowest rotational levels (rotational quantum numbers $j_{\mathrm{AP}}<8$ ) and practically all molecules were in the vibrational and electronic ground state. In the cold and collisionless environment of the molecular beam, interconversion between the conformers did not occur. After passing two skimmers, the molecular beam entered an electrostatic deflector consisting of a pair of $15 \mathrm{~cm}$ long electrodes to which potential differences in the range of 5 to $13 \mathrm{kV}$ were applied (26). 
The shape of the electrodes was designed to generate a strong inhomogeneous electric field with a nearly constant gradient along the $y$ axis as depicted in Fig. 1. For both conformers, the Stark energies of all populated rotational levels decrease with increasing field strength. The molecules are, therefore, deflected towards regions of high electric field in the deflector (20). Because the electric dipole moment is considerably larger for cis-AP than for trans-AP (25), the cis species is more strongly deflected than the trans species resulting in a spatial separation of the two conformers in the beam (20).

Fig. 2 A shows density profiles $n_{\text {cis }}(y)$ and $n_{\text {trans }}(y)$ for the deflected cis- and trans-AP molecules, respectively. The profiles were obtained by measuring the ion signal produced by conformer-selective resonance-enhanced multiphoton ionization (REMPI) (20) as a function of the vertical deflection coordinate when applying a potential difference of $V_{\text {defl }}=7.5 \mathrm{kV}$ to the deflector electrodes. The molecular beam assembly was tilted incrementally in the vertical direction in order to scan the deflected molecular beam over the position of the fixed ionization laser spot $82 \mathrm{~cm}$ downstream from the exit of the deflector. The deflection coordinate $y$ in Fig. 2 is defined as the vertical displacement of the center of the nominally undeflected beam from the interaction point. The dashed lines represent the results of Monte-Carlo trajectory simulations. In the calculations, the rotational temperature of the molecules and one global scaling factor were adjusted to optimally reproduce the experimental data. At low deflection coordinates the trans-conformation dominates, whereas at the highest deflection coordinates only the cis-conformer is present.

For the reaction experiments, the REMPI spectrometer was replaced by a linear quadrupole ion trap for the generation of Coulomb crystals of laser-cooled $\mathrm{Ca}^{+}$ions (4), see Fig. 1. The Coulomb crystals consisting of typically 700 ions were imaged by a camera sampling the atomic fluorescence generated by the laser cooling of the ions. The $\mathrm{Ca}^{+}$ions were exposed to AP molecules from the deflected molecular beam. Because the AP molecules in the reaction volume 
were replenished with each gas pulse, their number density was essentially constant over the measurement time. Thus, pseudo-first-order reaction rate constants could be determined from the decrease of the number of $\mathrm{Ca}^{+}$ions in the crystals (27). Product ions formed in the reaction remained confined in the trap. They were sympathetically cooled by the interaction with the remaining laser-cooled $\mathrm{Ca}^{+}$ions and localized at the edges of the Coulomb crystals (4). These ions did not fluoresce and were only indirectly visible in the images through a characteristic flattening of the $\mathrm{Ca}^{+}$crystal edges (28) as shown in Fig. 1.

The products of the reaction were analyzed using resonant-excitation mass spectrometry of the ions in the trap, suggesting $\mathrm{CaOH}^{+}$or $\mathrm{CaNH}_{2}^{+}$ions and the corresponding 3-aminophenyl or 3-hydroxiphenyl radicals as the primary reaction products. In the experiment, the $(4 s)^{2} S_{1 / 2}$, $(4 p)^{2} P_{1 / 2}$, and $(3 d)^{2} D_{3 / 2}$ states of $\mathrm{Ca}^{+}$were populated as a consequence of the laser cooling. The rate constant for reactions out of the excited $(4 p)^{2} P_{1 / 2}$ state was found to be two to three orders of magnitude larger than the rate coefficients for reactions out of the two other states so that this process dominated the effective rates measured in the present study. In the following, we focus on the $\mathrm{Ca}^{+}(4 p)+\mathrm{AP}$ reaction and all second-order rate constants quoted below refer to this particular channel.

Fig. 2 B shows the total effective pseudo-first-order rate constant $k_{1, \text { total }}$ as a function of the deflection coordinate $y$. The measured rate constant profile $k_{1, \text { total }}(y)$ reflects both the density distribution of the conformers in the deflected molecular beam as well as the conformer-specific second-order rate constants $k_{2, \text { cis }}$ and $k_{2, \text { trans }}$ for the reactions of cis-AP and trans-AP, respectively, with $\mathrm{Ca}^{+}(4 p)$ :

$$
k_{1, \text { total }}(y)=k_{2, \text { cis }} p_{4 p} n_{\text {cis }}(y)+k_{2, \text { trans }} p_{4 p} n_{\text {trans }}(y)
$$

$p_{4 p}$ is the population in the $\mathrm{Ca}^{+}(4 p)$ state. $k_{2, \text { cis }}$ and $k_{2, \text { trans }}$ were determined from a global fit of Eq. 1 to reaction-rate profiles obtained at deflector voltages $V_{\text {defl }}=5,7.5,10$, and $13 \mathrm{kV}$. 
The fit yielded the rate constants $k_{2, \text { cis }}=(3.2 \pm 1.3) \times 10^{-9} \mathrm{~cm}^{3} \mathrm{~s}^{-1}, k_{2, \text { trans }}=(1.5 \pm 0.6) \times$ $10^{-9} \mathrm{~cm}^{3} \mathrm{~s}^{-1}$ and the ratio $k_{2, \text { cis }} / k_{2, \text { trans }}=2.1 \pm 0.5$ within a $95 \%$ confidence interval. Fig. $2 \mathrm{C}$ shows the total second-order rate constant $k_{2 \text {,total }}(y)=x_{\text {cis }}(y) k_{2, c i s}+x_{\text {trans }}(y) k_{2, \text { trans }}$ obtained from the fit, where $x_{c i s}(y)$ and $x_{\text {trans }}(y)$ denote the mole fractions of cis-AP and trans-AP in the molecular beam, respectively. This plot highlights the change of the reaction rate as the beam composition evolves from trans to cis.

The rate constants $k_{2, \text { cis }}$ and $k_{2, \text { trans }}$ are of similar magnitude as the ones typically obtained for ion-molecule reactions with capture-limited kinetics (29). In these cases, the reaction rates are dominated by long-range intermolecular interactions in the entrance channel and are independent of the details of the short-range reaction mechanism. The rate constants can then appropriately be modeled using adiabatic capture models (30-32). These models assume that the reaction happens with unit probability if the collision energy exceeds the height of the centrifugal barrier in the entrance channel at a given value of the total angular momentum. Fig. $3 \mathrm{~A}$ and B show the centrifugally corrected long-range potentials for different values of the total angular momentum quantum number $J$ in the entrance channel of the reaction of $\mathrm{Ca}^{+}$with cisand trans-AP, respectively. The potentials have been constructed from the charge-dipole and the charge-induced dipole interactions, which represent the dominant long-range forces relevant for the collisions (31).

Due to the larger dipole moment of the cis-conformer, the interaction potential is more strongly attractive for cis-AP than for trans-AP. This can directly be seen from a comparison of the $J=0$ potentials of the cis- and trans-conformers in Fig. $3 \mathrm{~A}$ and $\mathrm{B}$, respectively. As a result, the centrifugal barrier is more strongly suppressed in the reaction with cis-AP so that reactive collisions can occur up to higher maximum values $J_{\max }$ of the total angular momentum quantum number $J$. At the collision energy of the experiment, we find $J_{\max }=417$ and $J_{\max }=342$ for the cis- and trans-conformers, respectively. Hence, in a classical picture the cis-conformer 
exhibits a larger maximum impact parameter $b_{\max }=J_{\max } / \mu v$ for the reaction (where $\mu$ is the reduced mass and $v$ the collision velocity) and, therefore, an increased reaction cross section $\sigma=\pi b_{\max }^{2}$. Averaging over all populated rotational states, the adiabatic capture model predicts the rate constants $k_{2, \text { cis }}=2.7 \times 10^{-9} \mathrm{~cm}^{3} \mathrm{~s}^{-1}$ and $k_{2, \text { trans }}=1.8 \times 10^{-9} \mathrm{~cm}^{3} \mathrm{~s}^{-1}$. The ratio of the theoretical rate constants is calculated to be $k_{2, \text { cis }} / k_{2, \text { trans }}=1.5$ in agreement with the experimental results. Moreover, the absolute values of these capture rate constants are in very good agreement with the experimentally obtained values. Thus, in the present case the increased reaction rate for the cis species can be traced back to its increased collision rate with $\mathrm{Ca}^{+}$. This effect results from conformation-dependent electrostatic properties of the molecules and the resulting differences in the long-range interaction potentials. Short-range conformational effects play only a minor role.

The isolation of individual conformers opens up new avenues to control the outcome of chemical reactions by selecting conformer-specific pathways that result in different product distributions. This capability has already been demonstrated in previous conformationally resolved photodissociation experiments $(15,16)$ and is now within reach for gas-phase reaction experiments.

The present advances have become possible through the combination of electrostatic conformer selection with highly sensitive Coulomb-crystal methods. We expect that the current methodology will benefit fundamental reaction-dynamics studies as well as the investigation of a wide range of ion-molecule reactions with relevance for catalysis and interstellar chemistry. Electrostatic conformer selection is a widely applicable technique whenever the conformers present in the molecular beam possess sufficiently different dipole moments. Even more advanced electric field manipulation techniques for the separation of individual chemical species $(19,33)$ or individual quantum states $(1,34)$ have been demonstrated. In addition, sympathetic cooling of ions is a near-universal technique that allows the generation of Coulomb 
crystals of a wide range of atomic and molecular species with simultaneous preparation of their internal quantum state $(4,35)$.

\section{References and Notes}

1. S. Y. T. van de Meerakker, H. L. Bethlem, N. Vanhaecke, G. Meijer, Chem. Rev. 112, 4828 (2012).

2. G. Quéméner, P. S. Julienne, Chem. Rev. 112, 4949 (2012).

3. F. Filsinger, G. Meijer, H. Stapelfeldt, H. N. Chapman, J. Küpper, Phys. Chem. Chem. Phys. 13, 2076 (2011).

4. S. Willitsch, Int. Rev. Phys. Chem. 31, 175 (2012).

5. J. J. Gilijamse, S. Hoekstra, S. Y. T. van de Meerakker, G. C. Groenenboom, G. Meijer, Science 313, 1617 (2006).

6. M. Kirste, et al., Science 338, 1060 (2012).

7. A. B. Henson, S. Gersten, Y. Shagam, J. Narevicius, E. Narevicius, Science 338, 234 (2012).

8. S. Ospelkaus, et al., Science 327, 853 (2010).

9. F. H. J. Hall, S. Willitsch, Phys. Rev. Lett. 109, 233202 (2012).

10. J. P. Simons, et al., Int. Rev. Phys. Chem. 24, 489 (2005).

11. M. S. de Vries, P. Hobza, Annu. Rev. Phys. Chem. 58, 585 (2007).

12. T. R. Rizzo, J. A. Stearns, O. V. Boyarkin, Int. Rev. Phys. Chem. 28, 481 (2009).

13. B. C. Dian, J. R. Clarkson, T. S. Zwier, Science 303, 1169 (2004).

14. B. C. Dian, G. G. Brown, K. O. Douglas, B. H. Pate, Science 320, 924 (2008).

15. S. T. Park, S. Y. Kim, M. S. Kim, Nature 415, 306 (2002).

16. M. H. Kim, L. Shen, H. Tao, T. J. Martinez, A. G. Suits, Science 315, 1561 (2007).

17. L. Khriachtchev, et al., J. Phys. Chem. A 113, 8143 (2009).

18. C. A. Taatjes, et al., Science 340, 177 (2013).

19. F. Filsinger, U. Erlekam, G. von Helden, J. Küpper, G. Meijer, Phys. Rev. Lett. 100, 133003 (2008).

20. F. Filsinger, et al., Angew. Chem. Int. Ed. 48, 6900 (2009).

21. K. Eller, H. Schwarz, Chem. Rev. 91, 1121 (1991).

22. H. Schwarz, Angew. Chem. Int. Ed. 50, 10096 (2011).

23. V. Ryzhov, R. C. Dunbar, J. Am. Chem. Soc. 121, 2259 (1999).

24. T. W. Robinson, H. G. Kjaergaard, S.-I. Ishiuchi, M. Shinozaki, M. Fujii, J. Phys. Chem. A 108, 4420 (2004).

25. F. Filsinger, K. Wohlfart, M. Schnell, J.-U. Grabow, J. Küpper, Phys. Chem. Chem. Phys. 10, 666 (2008).

26. F. Filsinger, et al., J. Chem. Phys. 131, 064309 (2009).

27. S. Willitsch, M. T. Bell, A. D. Gingell, S. R. Procter, T. P. Softley, Phys. Rev. Lett. 100, 043203 (2008).

28. S. Willitsch, M. T. Bell, A. D. Gingell, T. P. Softley, Phys. Chem. Chem. Phys. 10, 7200 (2008). 
29. B. R. Rowe, J. B. Marquette, Int. J. Mass Spectrom. Ion Proc. 80, 239 (1987).

30. D. C. Clary, Mol. Phys. 54, 605 (1985).

31. T. Stoecklin, D. C. Clary, A. Palma, J. Chem. Soc. Faraday Trans. 88, 901 (1992).

32. J. Troe, J. Chem. Phys. 87, 2773 (1987).

33. S. Trippel, et al., Phys. Rev. A 86, 033202 (2012).

34. J. H. Nielsen, et al., Phys. Chem. Chem. Phys. 13, 18971 (2011).

35. X. Tong, A. H. Winney, S. Willitsch, Phys. Rev. Lett. 105, 143001 (2010).

Acknowledgements: This work has been supported by the excellence cluster "The Hamburg Center for Ultrafast Imaging - Structure, Dynamics and Control of Matter at the Atomic Scale" of the Deutsche Forschungsgemeinschaft, the Swiss National Science Foundation grant. nr. PP00P2_140834, and the University of Basel. 


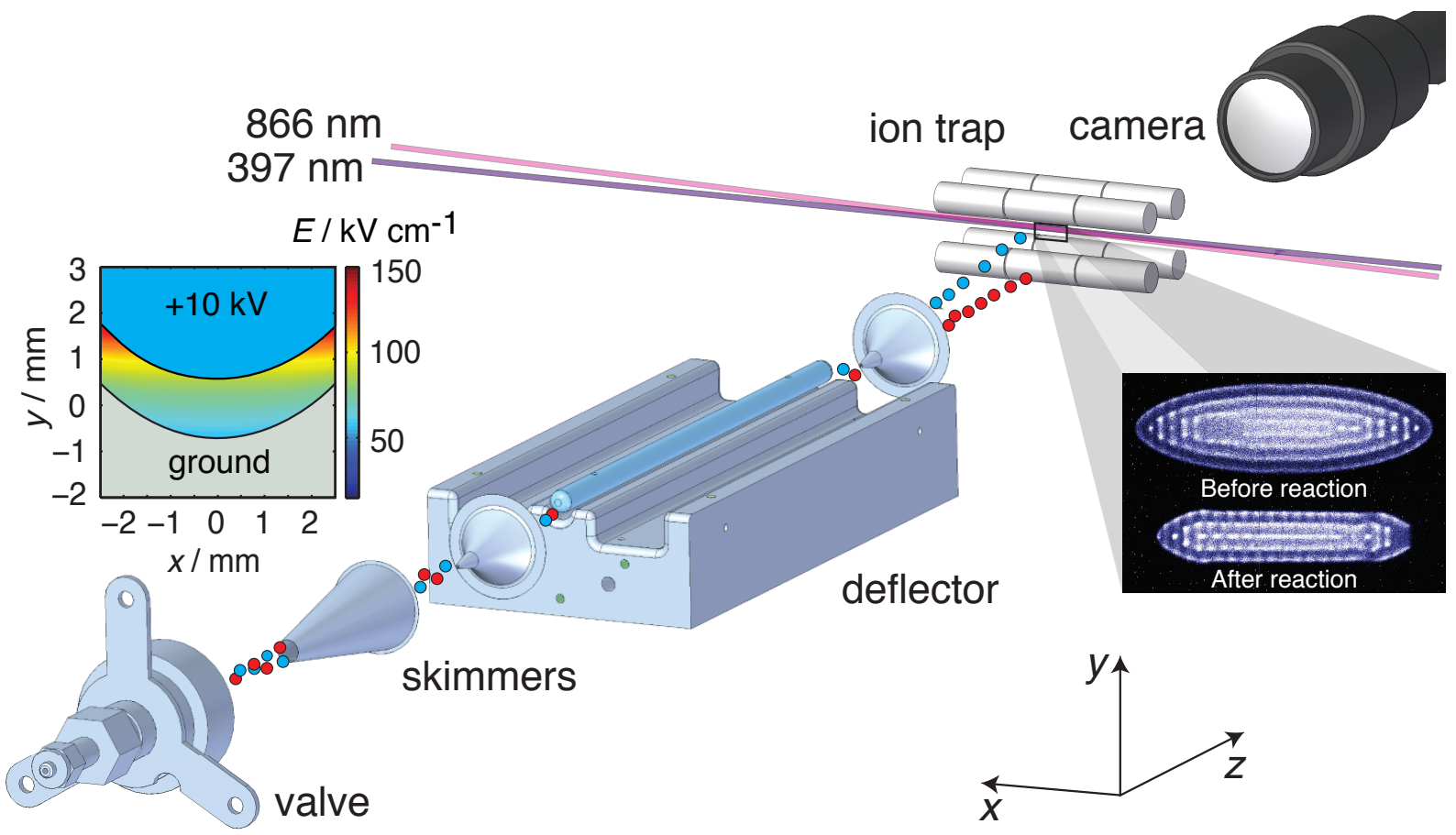

Figure 1: Experimental setup for studying conformer-selected chemical reactions. cis- (blue spheres) and trans- (red spheres) 3-aminophenol (AP) molecules are entrained in a molecular beam. The two conformers are deflected to different extents in the inhomogeneous electric field $E$ (left inset) of an electrostatic deflector. As a consequence, the molecular beam is split into different conformational components. The individual conformers are subsequently aimed at a stationary reaction target comprising a Coulomb crystal of laser-cooled $\mathrm{Ca}^{+}$ions in a trap (right inset). Conformer-specific rate constants are determined by monitoring the removal of $\mathrm{Ca}^{+}$ions from the Coulomb crystal as a function of the reaction time. $866 \mathrm{~nm}$ and $397 \mathrm{~nm}$ refer to the wavelengths of the $\mathrm{Ca}^{+}$cooling laser beams. 

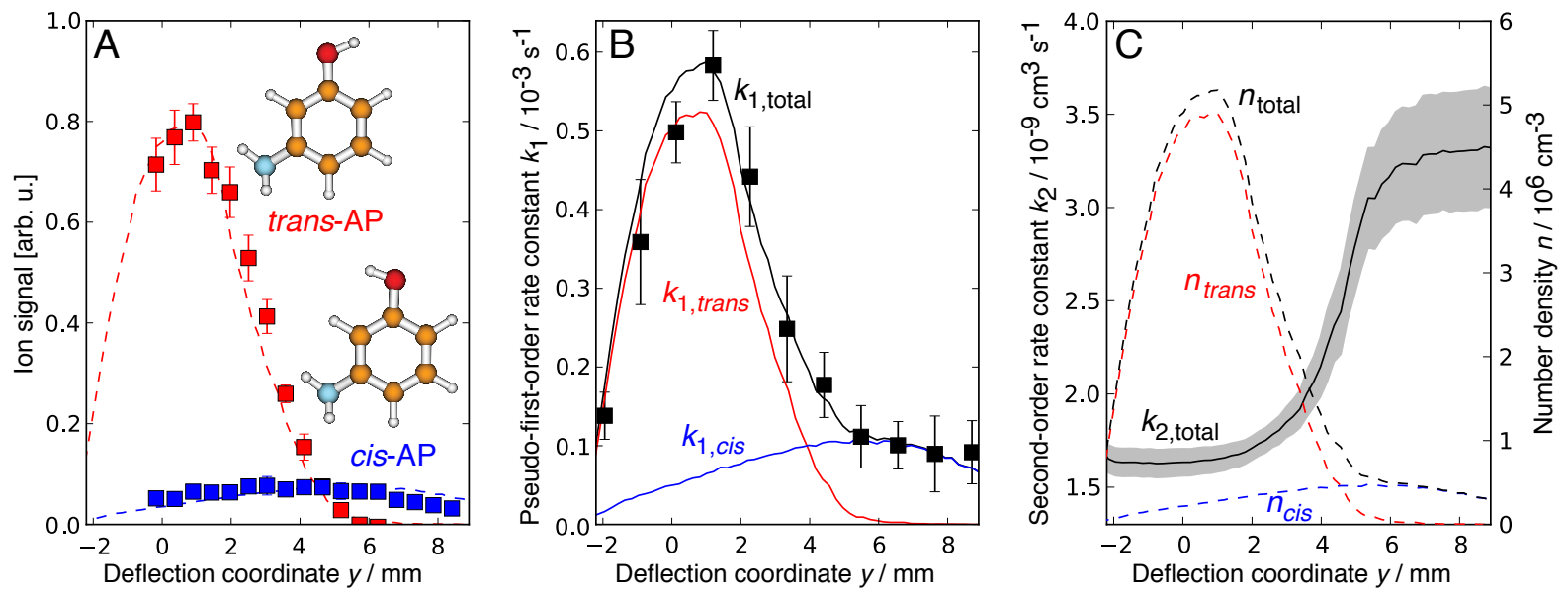

Figure 2: Reaction kinetics. (A) Density profiles of a deflected beam of cis- and trans-AP (deflector voltage $V_{\text {defl }}=7.5 \mathrm{kV}$ ) measured by conformer-specific multi-photon ionization as a function of the molecular-beam deflection coordinate $y$. Squares: experimental data, dashed lines: Monte-Carlo trajectory simulations. (B) Conformer-specific (red and blue) and total (black) pseudo-first-order rate constants $k_{1}$ for the reaction $\mathrm{Ca}^{+}+$cis-/trans-AP as a function of the beam deflection coordinate $y$ at $V_{\text {defl }}=7.5 \mathrm{kV}$. (C) Total bimolecular rate constant $k_{2, \text { total }}(y)$ (black solid line) illustrating the increase in chemical reactivity as the predominant beam component changes from the trans to the cis conformer. Dashed lines: number densities of the two conformers. The data points represent the statistical averages of 1000 laser shots in (A) and a minimum of four reaction measurements in (B). Error bars indicate the corresponding $95 \%$ confidence intervals. The gray area in (C) represents the $95 \%$ confidence interval of $k_{2, \text { total }}$. 


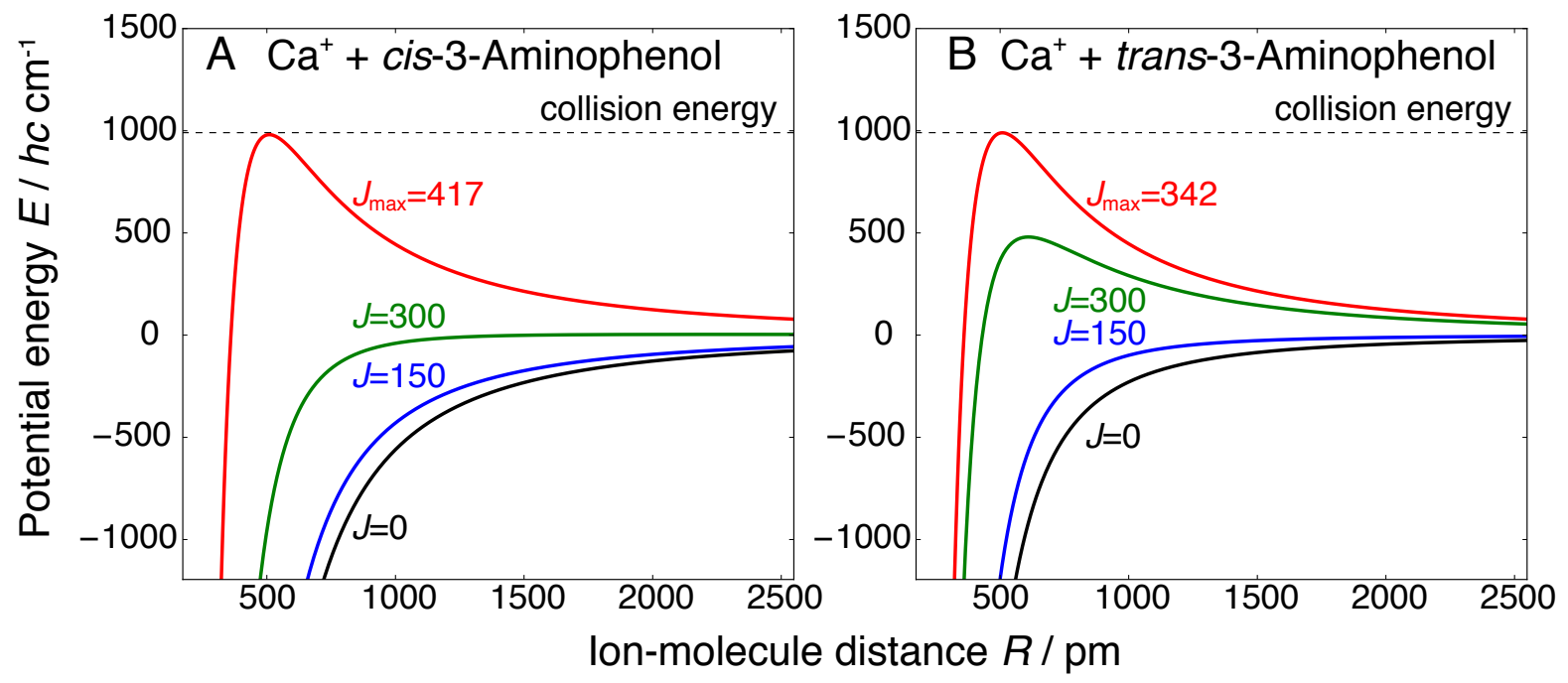

Figure 3: Centrifugally corrected potential energy curves for rotationless (A) cis- and (B) transAP molecules reacting with $\mathrm{Ca}^{+}$. 